Bundesgesundheitsbl 2022 $65: 159-169$ https://doi.org/10.1007/s00103-021-03473-z Eingegangen: 12. August 2021

Angenommen: 3. Dezember 2021

Online publiziert: 27. Dezember 2021

c c Der/die Autor(en) 2021

Annika Brodzinski ${ }^{1}$. Angela Neumeyer-Gromen ${ }^{2} \cdot$ Sandra Dudareva $^{3}$. Ruth Zimmermann ${ }^{3}$. Ute Latza ${ }^{4}$. Viviane Bremer ${ }^{3}$. Christina Poethko-Müller ${ }^{5}$ ${ }^{1}$ Institut für Hygiene und Umweltmedizin, Charité - Universitätsmedizin Berlin, Berlin, Deutschland; ${ }^{2}$ Deutsche Krankenhausgesellschaft e. V. (DKG), Berlin, Deutschland; ${ }^{3}$ Abteilung für Infektionsepidemiologie, Robert Koch-Institut, Berlin, Deutschland; ${ }^{4}$ Fachbereich Arbeit und Gesundheit, Bundesanstalt für Arbeitsschutz und Arbeitsmedizin BAuA, Berlin, Deutschland; ${ }^{5}$ Abteilung für Epidemiologie und Gesundheitsmonitoring, FG 25 Körperliche Gesundheit, Robert Koch-Institut, Berlin, Deutschland

\title{
Hepatitis-B-Virus-Infektionen und impfinduzierte Immunität: die Rolle von soziodemo- grafischen Determinanten
}

\author{
Ergebnisse der "Studie zur Gesundheit \\ Erwachsener in Deutschland" (DEGS1, \\ 2008-2011)
}

\section{Zusatzmaterial online}

Zusätzliche Informationen sind in der Online-Version dieses Artikels (https://doi. org/10.1007/s00103-021-03473-z) enthalten.

\section{Einleitung}

Die Hepatitis-B-Virus-(HBV-)Infektion ist eine der häufigsten Infektionskrankheiten weltweit, weist aber in der Prävalenz deutliche regionale Unterschiede auf [1]. Sie ist zudem ein bedeutender Risikofaktor für die Entwicklung einer Leberzirrhose und eines hepatozellulären Karzinoms und verursachte im Jahr 2015 weltweit ca. 887.000 Todesfälle [1]. Die Weltgesundheitsorganisation (WHO) hat ihren Mitgliedsstaaten daher zum Ziel gesetzt, die virale Hepatitis als Bedrohung für die öffentliche Gesundheit bis 2030 zu eliminieren. Eine 2016 verabschiedete Strategie sieht vor, dass Neuinfektionen und Mortalität durch weltweite synergistische Interventionen, wie z.B. eine Erhöhung der Impfquoten, eingedämmt werden [2]. Deutschlands nationale Strategie beinhaltet u. a. die Entwicklung von Präventions-, Beratungs-, Test- und Versorgungsangeboten, die sich an spe- zifischen Bedarfen von Risikogruppen, wie z.B. Jugendlichen, Migrantinnen und Migranten sowie Menschen mit beruflichem Expositionsrisiko, orientieren [3].

In der „Studie zur Gesundheit Erwachsener in Deutschland (DEGS1, 2008-2011)“ betrug die Prävalenz der aktiven (d.h. akuten oder chronischen) HBV-Infektion, definiert durch ein positives Hepatitis-B-Surface-Antigen (HBsAg), bei Erwachsenen im Alter von 18-79 Jahren 0,3 \% [4]. Deutschland zählt damit zu den Niedrigprävalenzregionen, in denen die Infektion typischerweise im jungen Erwachsenenalter durch Risikoverhalten wie Drogengebrauch oder sexuelles Risikoverhalten erworben wird [5, 6]. Antikörper gegen das Hepatitis-BCore-Antigen (Anti-HBc) stehen für eine stattgehabte oder aktive HBV-Infektion und waren bei 5,1\% der Allgemeinbevölkerung nachweisbar [4]. Verglichen mit den Ergebnissen des „Bundesgesundheitssurveys 98“ (BGS98, 1997-1999) war die Anti-HBc-Prävalenz von 8,7\% auf 5,1\% gesunken [4].

Die Prävention der HBV-Infektion beinhaltet z.B. das Screening von Personen, die Blut und Plasma spenden, die Förderung von Safer Sex und die Reduktion von Gefahren beim intravenösen Drogenkonsum [1]. Außerdem ist seit Anfang der 1980er-Jahre eine sichere und effektive Impfung gegen HBV verfügbar. Nachdem die Hepatitis-B-Impfung in Deutschland initial nur bei Risikogruppen wie medizinischem Personal oder Dialysepatienten eingesetzt wurde, empfahl die Ständige Impfkommission (STIKO) 1995 zusätzlich die Impfung von allen Kindern und Jugendlichen [7]. Die HBV-Impfung ist seitdem eine Standardimpfung für Säuglinge und wird bis zum 18. Geburtstag als Nachholimpfung angeboten [7]. In den bevölkerungsrepräsentativen Surveys BGS98 und DEGS1 stieg die Prävalenz der impfinduzierten Immunität, definiert durch alleinigen Nachweis von Antikörpern gegen das Hepatitis-B-Surface-Antigen (Anti-HBs), bei Erwachsenen im Alter von 18-79 Jahren von 4,3\% (1997-1999) auf 22,9\% (2008-2011; [4]). Querschnittsuntersuchungen in Risikogruppen zeigen jedoch eine erhöhte Prävalenz stattgehabter HBV-Infektionen bei gleichzeitig bestehenden Impflücken [8,9]. Auch die für die Jahre 2008 bis 2011 errech- 
Tab. 1 Klinische Interpretation der Ergebnisse der HBV-Serologie und Einteilung in Kategorien. (Adaptiert nach [4])

\begin{tabular}{|c|c|c|c|c|}
\hline \multicolumn{3}{|c|}{$\begin{array}{l}\text { Ergebnisse der HBV- } \\
\text { Serologie }\end{array}$} & \multirow[t]{2}{*}{ Klinische Interpretation } & \multirow[t]{2}{*}{ Kategorie } \\
\hline $\begin{array}{l}\text { Anti- } \\
\text { HBC }\end{array}$ & $\begin{array}{l}\text { Anti- } \\
\text { HBs }\end{array}$ & HBsAg & & \\
\hline+ & + & a & Erworbene Immunität gegen HBV nach einer Exposition & \multirow{3}{*}{$\begin{array}{l}\text { Stattgehabte } \\
\text { HBV-Infektion }\end{array}$} \\
\hline+ & - & + & Aktuell vorliegende HBV-Infektion (akut oder chronisch) & \\
\hline+ & - & - & $\begin{array}{l}\text { „Ausgeheilte“ oder okkulte HBV-Infektion (mit oder ohne } \\
\text { Immunität gegen HBV und der Möglichkeit der Reaktivie- } \\
\text { rung) }\end{array}$ & \\
\hline- & + & - & Erworbene Immunität gegen HBV durch eine Impfung & $\begin{array}{l}\text { Impfinduzierte } \\
\text { Immunität } \\
\text { gegen HBV }\end{array}$ \\
\hline- & - & - & $\begin{array}{l}\text { Kein Anhalt für eine stattgehabte Exposition gegenüber } \\
\text { HBV oder eine Impfung }\end{array}$ & $\begin{array}{l}\text { Suszeptibilität } \\
\text { gegenüber } \\
\text { HBV }\end{array}$ \\
\hline \multicolumn{5}{|c|}{$\begin{array}{l}\text { Ergebnisse der HBV-Serologie: +: reaktiv; -: negativ } \\
\text { HBV Hepatitis-B-Virus } \\
\text { a Keine Untersuchung des HBsAg, wenn Anti-HBC und Anti-HBs reaktiv waren }\end{array}$} \\
\hline
\end{tabular}

nete Inzidenz von $0,9-1,0$ gemeldeten Hepatitis-B-Fällen pro 100.000 Einwohnerinnen und Einwohner (EW) spricht für eine fortlaufende HBV-Transmission in Deutschland [10]. Eine zielgerichtete Prävention ist daher erforderlich, um besonders gefährdete Personen zu erreichen.

Übereinstimmend mit anderen bevölkerungsrepräsentativen Studien [11-15] ergab eine erste Analyse der DEGS1-Daten, dass die HBV-Durchseuchung mit dem Alter stieg und die Wahrscheinlichkeit für das Vorliegen einer impfinduzierten Immunität bei jüngeren Teilnehmenden und Frauen erhöht war [4]. Die HBVInfektion war außerdem mit einem niedrigeren und die impfinduzierte Immunität mit einem höheren sozioökonomischen Status (SES) assoziiert, einem Index, der sich aus Einkommen, schulischer und beruflicher Bildung sowie beruflicher Stellung zusammensetzt $[4,16]$. Internationale bevölkerungsbasierte Studien berichteten außerdem, dass HBV-Infektion und-Impfung mit Bildungsstand, Einkommen, Migrationsstatus und Gemeindegröße assoziiert waren [11-15, 17, 18]. Diese Determinanten wurden bislang in DEGS1 nicht differenziert untersucht; ihre Auswertung kann aber zusätzliche Erkenntnisse für die Verbesserung der HBV-Prävention liefern. Ziel dieser Studie war es daher, die Assoziationen der HBV-Infektion und -Impfung mit diesen Determinanten in der deutschen
Allgemeinbevölkerung detailliert zu analysieren.

\section{Methoden}

\section{Studiendesign}

Die Zielpopulation von DEGS1 waren Erwachsene im Alter von 18-79 Jahren, die in Deutschland lebten und nicht in Institutionen, wie z. B. Flüchtlingsunterkünften, untergebracht waren [19].

Die Stichprobe umfasste ehemalige Teilnehmende des BGS98 (Response $64 \%$ ) und eine neu gezogene zweistufige, geschichtete Klumpenstichprobe (Response 42\%) [19]. In der neu gezogenen Stichprobe ( $n=11.008$ Personen) wurden 275 Personen aufgrund erheblicher Sprachbarrieren ausgeschlossen [19, 20]. Konzept und Design von DEGS1 sind an anderer Stelle ausführlich beschrieben [19-21]. Die Erhebungen fanden zwischen 2008 und 2011 an 180 Studienorten statt und umfassten u.a. Selbstausfüllfragebögen, Interviews und Blutuntersuchungen. Für Teilnehmende, die den Selbstausfüllfragebogen nicht vollständig bearbeiten konnten, wurde eine gekürzte Version vorgehalten. In der Kurzversion fehlten u.a. Fragen zu Krankenversicherung, Migrationsgeneration und Beruf. Fragebögen in weiteren Sprachen (Türkisch, Serbokroatisch, Russisch und Englisch) wurden Teilnehmenden angeboten, die zwar Deutsch sprechen, aber nicht ausreichend lesen und schreiben konnten.

\section{HBV-Serologie}

Die Ergebnisse der HBV-Serologie wurden in 3 Kategorien unterteilt: 1) stattgehabte/aktive "HBV-Infektion“, definiert durch ein positives Anti-HBc, 2), ,impfinduzierte Immunität", definiert durch einen Anti-HBs-Spiegel $\geq 10 \mathrm{IU} / \mathrm{l}$ ohne Nachweis anderer HBV-Marker, und 3) „Suszeptibilität gegenüber HBV“, definiert durch das Fehlen von HBVMarkern (• Tab. 1). Das serologische Testverfahren wurde an anderer Stelle ausführlich beschrieben [4].

\section{Unabhängige Variablen}

Zur Schätzung der Prävalenzen wurden die Geburtsjahrgänge in 4-JahresKohorten unterteilt. Aufgrund kleiner Fallzahlen wurden die Geburtsjahrgänge 1989-1993 sowie 1928-1936 zusammengefasst. Für die logistische Regression wurden die Teilnehmenden in 3 Altersgruppen unterteilt: junge Erwachsene, die unter die Impfempfehlung für Kinder und Jugendliche fielen (18-33 Jahre), Erwachsene im mittleren Alter (34-64 Jahre) und Erwachsene im höheren Alter ( $\geq 65$ Jahre).

Das Einkommen wurde als bedarfsgewichtetes Haushaltsnettoeinkommen (Nettoäquivalenzeinkommen) angegeben und fehlende Werte durch ein Regressionsmodell imputiert [16]. Das Einkommen wurde in 3 Gruppen unterteilt: $\leq 60 \%,>60 \%$ bis $150 \%$ und $>150 \%$ des medianen Nettoäquivalenzeinkommens von privaten Haushalten in Deutschland [22]. Für den Studienzeitraum wurde für die Jahre 2007 bis 2010 ein Nettoäquivalenzeinkommen von $1558 € /$ Monat zugrunde gelegt [23] und die folgenden Kategorien wurden abgeleitet: "niedriges" ( $\leq 935 € /$ Monat $)$, "mittleres" (936-2337€/Monat) und "hohes Einkommen“ ( $\geq 2338 € /$ Monat).

Der formale Bildungsstand wurde in Anlehnung an die CASMIN-Klassifikation („Comparative Analyses of Social Mobility in Industrial Nations") erfasst, die Schulbildung und berufliche Qualifikation berücksichtigt $[16,24]$. Der 
Bundesgesundheitsbl 2022 ·65:159-169 https://doi.org/10.1007/s00103-021-03473-z

(c) Der/die Autor(en) 2021

\section{A. Brodzinski · A. Neumeyer-Gromen · S. Dudareva · R. Zimmermann · U. Latza · V. Bremer · C. Poethko-Müller}

\section{Hepatitis-B-Virus-Infektionen und impfinduzierte Immunität: die Rolle von soziodemografischen Determinanten. Ergebnisse der „Studie zur Gesundheit Erwachsener in Deutschland“ (DEGS1, 2008-2011)}

\section{Zusammenfassung}

Hintergrund und Ziel. Trotz niedriger Prävalenz der Hepatitis-B-Virus-(HBV-)Infektion in Deutschland ist es wichtig, vulnerable Gruppen und Ansatzpunkte für die Prävention zu identifizieren. In ersten Analysen der "Studie zur Gesundheit Erwachsener in Deutschland" (DEGS1, 2008-2011) waren HBV-Infektion und -Impfung mit sozidemografischen Determinanten assoziiert. In dieser Arbeit werden die Ergebnisse im Detail untersucht. Material und Methoden. In DEGS1 lag für 7046 Teilnehmende (Alter: 18-79 Jahre) eine HBV-Serologie vor. Die stattgehabte HBV-Infektion war durch Antikörper gegen das Hepatitis-B-Core-Antigen (Anti-HBc) definiert, die impfinduzierte Immunität durch alleinigen Nachweis von Antikörpern gegen das Hepatitis-B-Surface-Antigen (Anti-HBs). Seroprävalenzen von HBV-Infektions- und -Impfstatus wurden geschlechtsstratifiziert geschätzt und Assoziationen mit Alter, Gemeindegröße, Einkommen, formaler Bildung, Krankenversicherung und Migrationsgeneration in logistischen Regressionen analysiert.

Ergebnisse. Die HBV-Infektion war bei Männern und Frauen unabhängig mit den Altersgruppen 34-64 und $\geq 65$ Jahre, erster Migrationsgeneration und Leben in größeren Gemeinden assoziiert, zudem bei Männern mit niedrigem Einkommen und bei Frauen mit niedriger Bildung. Die impfinduzierte
Immunität war bei Männern und Frauen unabhängig mit den Altersgruppen 18-33 und 34-64 Jahre, mittlerer und hoher Bildung und hohem Einkommen assoziiert, darüber hinaus bei Männern mit mittlerem Einkommen und privater Krankenversicherung und bei Frauen mit fehlendem Migrationshintergrund. Diskussion. Die Berücksichtigung von Migrationsstatus, Einkommen und Bildung könnte zur zielgenauen Ausrichtung der HBVPrävention beitragen.

\section{Schlüsselwörter}

Hepatitis B · Epidemiologie · Impfung Prävalenz · Deutschland

\section{Hepatitis B virus infection and vaccine-induced immunity: the role of sociodemographic determinants. Results of the study "German Health Interview and Examination Survey for Adults" (DEGS1, 2008-2011)}

\begin{abstract}
Background and objective. Even though the prevalence of hepatitis B virus (HBV) infection in Germany is low, it is important to identify vulnerable groups and targeted approaches for infection prevention. Previous analyses from the "German Health Interview and Examination Survey for Adults" (DEGS1, 2008-2011) have shown that HBV infections and vaccination are associated with sociodemographic determinants. This paper examines the results in detail.

Materials and methods. In the DEGS1, HBV serology was available for 7046 participants aged $18-79$ years. HBV infection was defined by antibodies to hepatitis $B$ core antigen (anti-
\end{abstract}

$\mathrm{HBc}$ ), vaccine-induced immunity by antibodies to hepatitis B surface antigen (anti-HBs) in the absence of other markers. Seroprevalences of HBV infection and vaccine-induced immunity were estimated stratified by sex, and associations with age, municipality size, income, formal education, health insurance and migration generation were analysed by logistic regression.

Results. In both sexes, HBV infection was independently associated with age groups 34-64 and $\geq 65$ years, first migrant generation and living in larger municipalities as well as low income in men and low education in women. Vaccine-induced immunity was independently associated with age groups 18-33 and 34-64 years, middle and high education and high income in both sexes, middle income and private health insurance in men and having no migration background in women.

Conclusions. HBV prevention measures should take into account migration status, income and education in order to focus prevention measures.

\section{Keywords}

Hepatitis B - Epidemiology · Vaccination . Prevalence - Germany
Bildungsstand wurde nach Brauns et al. in 3 Kategorien unterteilt [25]: „niedrig“ (kein Abschluss, Hauptschulabschluss und/ohne berufliche Ausbildung), ,mittel“ (Mittlere Reife, Fachhochschulreife oder Abitur, jeweils und/ohne berufliche Ausbildung) und "hoch" (Fachhochschulabschluss, Hochschulabschluss).

Der Krankenversicherungsstatus war in „gesetzlich“, „privat“ und „,andere“ kategorisiert. Personen der ersten Migrationsgeneration waren im Ausland ge- boren, während Personen der zweiten Migrationsgeneration in Deutschland geboren waren, aber mindestens einen im Ausland geborenen Elternteil hatten. Die Teilnehmenden sollten ihren aktuellen Beruf oder, falls sie nicht erwerbstätig waren, ihren zuletzt ausgeübten Beruf angeben. Die Kategorisierung der Berufe war an die „Klassifikation der Berufe 2010“ der Bundesagentur für Arbeit angelehnt [26]. Die für diese Analyse ausge- wählten Berufe des Gesundheitswesens sind im Onlinematerial aufgeführt.

Die Gemeindegröße am 31.12.2006 wurde in ländlich $(<5000 \mathrm{EW})$, kleinstädtisch $(5000$ bis $<20.000 \mathrm{EW})$, mittelstädtisch $(20.000$ bis $<100.000 \mathrm{EW})$ und großstädtisch $(\geq 100.000 \mathrm{EW})$ unterteilt.

\section{Statistische Auswertung}

Die Auswertung erfolgte mit statistischen Verfahren für komplexe Stichproben in 
Tab. 2 Soziodemografische Charakteristika der Studienpopulation

Männer Frauen

\begin{tabular}{|c|c|c|c|c|}
\hline & Anzahla & $\begin{array}{l}\text { Gewichtete Anteile in \% } \\
\text { (95\%-KI) }\end{array}$ & Anzahla & $\begin{array}{l}\text { Gewichtete Anteile in \% } \\
\text { (95\%-KI) }\end{array}$ \\
\hline Studienpopulation & 3376 & - & - & - \\
\hline \multicolumn{5}{|l|}{ Alter in Jahren } \\
\hline $18-33$ & 657 & $24,5(22,8-26,3)$ & 694 & $23,9(22,3-25,6)$ \\
\hline $34-64$ & 1805 & $57,4(55,5-59,2)$ & 2062 & $55,3(53,4-57,2)$ \\
\hline$\geq 65$ & 914 & $18,1(17,0-19,4)$ & 914 & $20,8(19,3-22,3)$ \\
\hline Fehlt & 0 & 0 & 0 & 0 \\
\hline \multicolumn{5}{|l|}{ Gemeindegröße } \\
\hline Ländlich & 651 & $16,0(10,9-23,0)$ & 642 & $14,7(9,8-21,4)$ \\
\hline Kleinstädtisch & 832 & $23,9(17,9-31,3)$ & 875 & $24,1(17,9-31,5)$ \\
\hline Mittelstädtisch & 958 & $28,8(22,1-36,5)$ & 1086 & $29,7(22,9-37,5)$ \\
\hline Großstädtisch & 935 & $31,3(24,4-39,1)$ & 1067 & $31,6(24,7-39,5)$ \\
\hline Fehlt & 0 & 0 & 0 & 0 \\
\hline \multicolumn{5}{|l|}{ Einkommen } \\
\hline Niedrig & 929 & $30,1(27,8-32,6)$ & 1075 & $32,2(30,2-34,2)$ \\
\hline Mittel & 1979 & $57,3(54,7-59,9)$ & 2199 & $58,1(56,0-60,2)$ \\
\hline Hoch & 468 & $12,6(11,0-14,4)$ & 396 & $9,8(8,4-11,3)$ \\
\hline Fehlt & 0 & 0 & 0 & 0 \\
\hline \multicolumn{5}{|l|}{ Bildungsstand } \\
\hline Niedrig & 1096 & $36,1(33,3-39,0)$ & 1195 & $37,4(35,0-39,9)$ \\
\hline Mittel & 1502 & $46,5(44,1-48,9)$ & 1889 & $49,6(47,3-51,9)$ \\
\hline Hoch & 754 & $16,5(14,9-18,4)$ & 563 & $12,0(10,4-13,9)$ \\
\hline Fehlt & 24 & $0,9(0,5-1,5)$ & 23 & $1,0(0,6-1,8)$ \\
\hline \multicolumn{5}{|l|}{ Krankenversicherung } \\
\hline Gesetzlich & 2765 & $81,9(80,0-83,7)$ & 3251 & $88,8(87,3-90,2)$ \\
\hline Privat & 492 & $14,0(12,5-15,8)$ & 348 & $8,6(7,4-10,1)$ \\
\hline Fehlt/andere & 119 & $4,1(3,2-5,2)$ & 71 & $2,5(1,9-3,4)$ \\
\hline \multicolumn{5}{|c|}{ Migrationsgeneration } \\
\hline Keine & 2783 & $77,3(74,4-79,9)$ & 3037 & $76,6(74,1-79,0)$ \\
\hline Erste & 277 & $12,2(10,3-14,3)$ & 321 & $13,2(11,3-15,4)$ \\
\hline Zweite und weitere & 198 & $6,5(5,4-7,9)$ & 205 & $6,3(5,2-7,6)$ \\
\hline Fehlt & 118 & $4,0(3,1-5,1)$ & 107 & $3,9(3,0-5,0)$ \\
\hline \multicolumn{5}{|c|}{ Letzter/aktueller Beruf } \\
\hline Gesundheitsberuf & 59 & $2,2(1,6-3,1)$ & 344 & $9,9(8,7-11,4)$ \\
\hline Anderer & 2900 & $89,4(87,3-91,2)$ & 2899 & $82,1(80,2-83,8)$ \\
\hline Fehlt & 226 & $8,4(6,8-10,3)$ & 222 & $8,0(6,6-9,6)$ \\
\hline
\end{tabular}

Die Ergebnisse werden als ungewichtete Fallzahlen und gewichtete Anteile mit 95\%-Konfidenzintervallen (KI) berichtet. Gewichtungsfaktoren wurden verwendet, um für Non-Response und Abweichungen von der Zielpopulation in Bezug auf Alter, Geschlecht, Region, Staatsangehörigkeit, Gemeindegröße und Bildungsstand zu korrigieren [19]. $n_{\text {ungewichtet }}=7046$

a Ungewichtete Anzahl

${ }^{b}$ Nur verfügbar bei Teilnehmenden, die jemals erwerbstätig waren (Männer: $n=3185$, Frauen: $n=3465$ )

Stata/SE Version 14. Gewichtungsfaktoren korrigierten für Non-Response und Abweichungen von der Bevölkerungsstruktur in Hinblick auf Alter, Geschlecht, Region, Staatsangehörigkeit, Gemeindegröße und Bildungsstand [21]. Da Frauen häufiger eine impfinduzierte Immunität gegen $\mathrm{HBV}$ aufwiesen als
Männer [4] und Risikofaktoren einer HBV-Infektion bei Männern und Frauen unterschiedlich verteilt sind [27, 28], wurden die Analysen nach Geschlecht stratifiziert. Die Prävalenzen der HBVInfektion und impfinduzierten Immunität wurden als gewichtete Anteile in Prozent mit 95\%-Konfidenzintervallen
(KI) geschätzt. Unterschiede wurden als statistisch signifikant angesehen, wenn sich die $95 \%$-Konfidenzintervalle nicht überlappten.

Assoziationen der HBV-Infektion und impfinduzierten Immunität mit $\mathrm{Al}$ ter, Einkommen, Bildung, Krankenversicherung, Gemeindegröße und Migrationsgeneration wurden in univariabler und multivariabler logistischer Regression analysiert. Es wurden Odds Ratios (OR) und 95\%-Konfidenzintervalle geschätzt. Durch schrittweise Vorwärtsselektion wurde das am besten passende Modell für die jeweiligen Outcomes ermittelt. Ergebnisse mit einem $p$-Wert $<0,05$ wurden als statistisch signifikant angesehen.

Analysen der DEGS1-Daten habengezeigt, dass bei Teilnehmenden mit Migrationshintergrund trotz des Einsatzes von Gewichtungsfaktoren keine Repräsentativität für Alter und Migrationsgeneration vorlag $[29,30]$. Analysen von Teilnehmenden mit Migrationshintergrund sollten deshalb nach Alter, Geschlecht, Migrationsgeneration und sozioökonomischem Status stratifiziert werden [29, 30]. Die Variable „Migrationsgeneration“ wurde daher nur in multivariablen Analysen untersucht.

Da Informationen zu einer „aktuellen/früheren Berufstätigkeit im Gesundheitswesen " nur bei Teilnehmenden erhoben wurde, die jemals erwerbstätig gewesen waren, war der Anteil fehlender Angaben relativ hoch ( $\bullet$ Tab. 2). Auch in der Kategorie „fehlende/andere Krankenversicherung" waren die Fallzahlen niedrig (•Tab. 2), sodass die Variable „Beruf“ und die Kategorie „fehlende/ andere Krankenversicherung " in den logistischen Regressionen nicht berücksichtigt wurden.

\section{Ergebnisse}

Insgesamt hatten 7115 Erwachsene im Alter von 18-79 Jahren die Befragung und den Untersuchungsteil absolviert. Ergebnisse der HBV-Serologie lagen für 7046 Teilnehmende (99\%) vor. Die soziodemografischen Charakteristika dieser Teilnehmenden sind in - Tab. 2 dargestellt. 


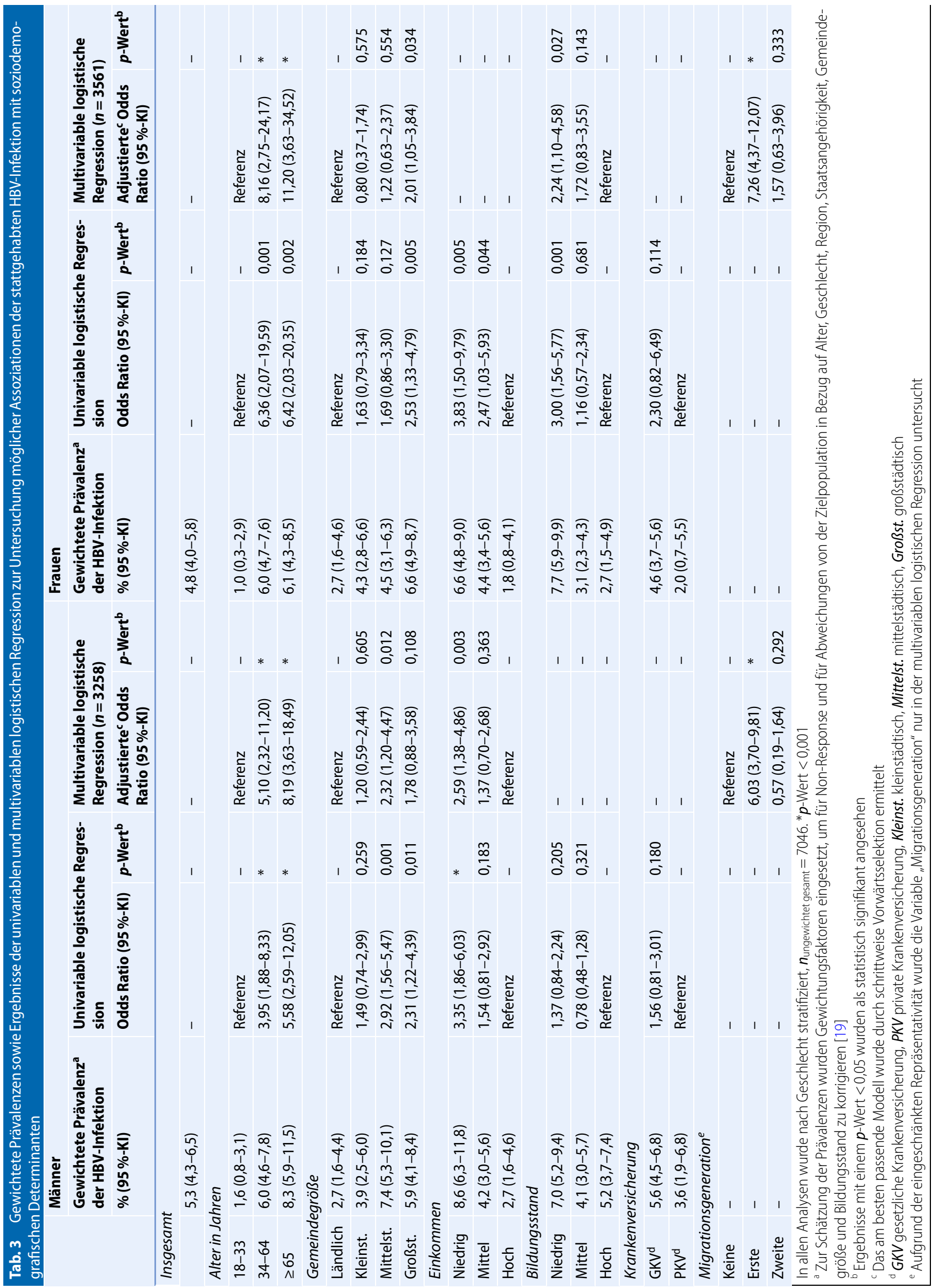




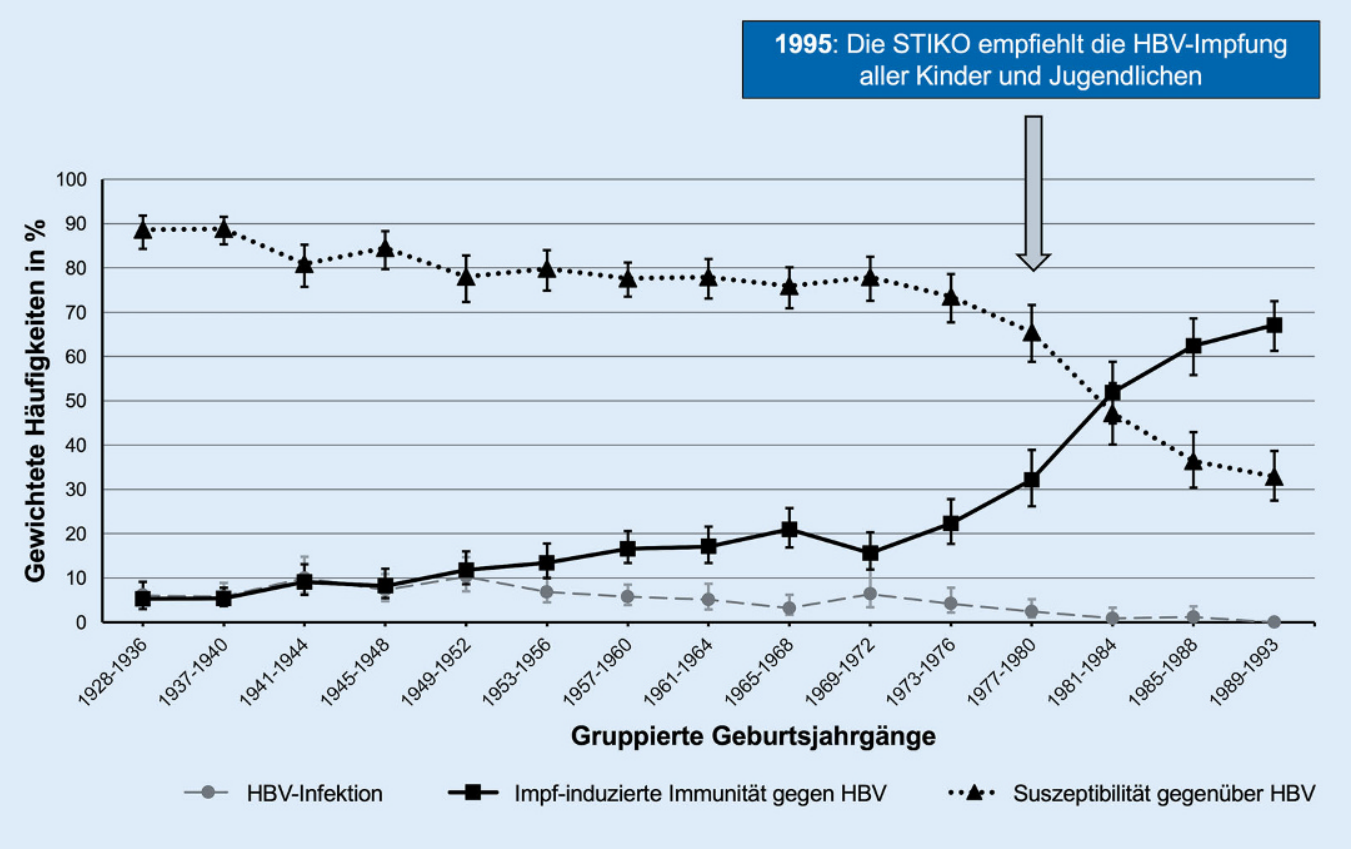

Abb. 1 Ergebnisse der Hepatitis-B-Virus(HBV)-Serologie nach gruppierten Geburtsjahren. Gewichtete Prävalenzen und $95 \%$-Konfidenzintervalle der HBVInfektion, impfinduzierten Immunität gegen HBV und Suszeptibilität für HBVnach gruppierten Geburtsjahren von 1928 bis 1993. (Eigene Abbildung).STIKOStändige Impfkommission

\section{HBV-Infektion}

Die Prävalenz der HBV-Infektion betrug 5,3\% (95\%-KI 4,3-6,5) bei Männern und 4,8\% (95\%-KI 4,0-5,8) bei Frauen.

Teilnehmende der Geburtsjahrgänge 1949-1952 wiesen die höchste Prävalenz von HBV-Infektionen auf (10,3\%; $95 \%$ KI 7,0-14,7), während in den Geburtsjahrgängen 1989-1993 keine stattgehabten HBV-Infektionen nachgewiesen wurden (• Abb. 1).

Bei Männern und Frauen waren die Prävalenzen von $\mathrm{HBV}$-Infektionen am höchsten in der Altersgruppe $\geq 65$ Jahre sowie bei Teilnehmenden mit niedrigem Einkommen und niedriger Bildung (- Tab. 3). Bezogen auf die Gemeindegröße waren die Prävalenzen von HBVInfektionen bei Männern in mittelgroßen Städten und bei Frauen in Großstädten am höchsten. Die Prävalenz von HBVInfektionen unterschied sich nicht zwischen den im Gesundheitswesen beschäftigten Teilnehmenden und jenen ohne eine solche Berufstätigkeit (• Tab.4).

In den multivariablen Analysen war eine HBV-Infektion bei Männern und Frauen statistisch signifikant mit höherer Altersgruppe (34-64, $\geq 65$ Jahre) und Zugehörigkeit zur ersten Migrationsgeneration assoziiert (• Tab. 3). Die HBV-Infektion war außerdem mit niedrigem Ein- kommen (Männer) bzw. niedriger Bildung (Frauen) assoziiert. Darüber hinaus zeigte sich eine statistisch signifikante Assoziation der HBV-Infektion mit dem Leben in mittelgroßen Städten (Männer) bzw. in Großstädten (Frauen).

\section{Impfinduzierte Immunität}

Die Prävalenz der impfinduzierten Immunität gegen $\mathrm{HBV}$ war bei Frauen signifikant höher $(25,8 \%$; $95 \%$-KI 23,8-27,8) als bei Männern (20,1\%; 95\%-KI 18,2-22,1).

Die Prävalenz der impfinduzierten Immunität war am niedrigsten bei den Geburtsjahrgängen 1928-1936 (6,1\%; 95\%-KI 4,1-9,2) und am höchsten bei den Geburtsjahrgängen 1989-1993 (67,1\%; 95\%-KI 61,3-72,5; • Abb. 1). Die Geburtsjahrgänge 1981-1984, die als erste unter die allgemeine Impfempfehlung für Kinder und Jugendliche gefallen waren, wiesen eine signifikant höhere Prävalenz der impfinduzierten Immunität auf als die vorhergehenden Geburtsjahrgänge 1977-1980 (51,9\%; $95 \%$-KI $44,9-58,8$ vs. $32,2 \%$; $95 \%$-KI 26,2-38,9).

Bei Männern und Frauen fanden sich die höchsten Prävalenzen der impfinduzierten Immunität in der Altersgruppe 18-33 Jahre sowie bei Teilnehmenden mit hohem Einkommen. Die höchsten Prävalenzen impfinduzierter Immunität zeigten sich außerdem bei Männern mit mittlerer und bei Frauen mit hoher Bildung (- Tab. 5). Männer mit privater Krankenversicherung (PKV) wiesen signifikant häufiger eine impfinduzierte Immunität auf als Männer mit gesetzlicher Krankenversicherung (GKV). Bei Teilnehmenden mit einem Beruf im Gesundheitswesen war die Prävalenz der impfinduzierten Immunität signifikant höher als bei Teilnehmenden mit anderen Berufen (•Tab. 4).

In den multivariablen Analysen für Männer und Frauen war die impfinduzierte Immunität statistisch signifikant mit jüngerer Altersgruppe (18-33, 34-64 Jahre), mittlerer und hoher Bildung sowie hohem Einkommen assoziiert (• Tab.5). Bei Männern zeigte sich außerdem eine statistisch signifikante Assoziation der impfinduzierten Immunität mit mittlerem Einkommen und privater Krankenversicherung. Die Chance, dass Frauen der ersten Migrationsgeneration eine impfinduzierte Immunität hatten, war signifikant geringer als bei Frauen ohne Migrationshintergrund. 


\begin{tabular}{|c|c|c|c|c|}
\hline & \multicolumn{2}{|c|}{$\begin{array}{l}\text { Beruf im Gesundheitswesen } \\
n=403\end{array}$} & \multicolumn{2}{|c|}{$\begin{array}{l}\text { Andere Berufe } \\
n=5799\end{array}$} \\
\hline & Anzahl & $\begin{array}{l}\text { Gewichtete Prävalenz } \\
\text { in } \%(95 \%-K I)\end{array}$ & Anzahl & $\begin{array}{l}\text { Gewichtete Prävalenz } \\
\text { in \% }(95 \%-K \mathrm{I})\end{array}$ \\
\hline HBV-Infektion & 21 & $4,1(2,5-6,7)$ & 234 & $4,3(3,6-5,1)$ \\
\hline $\begin{array}{l}\text { Impfinduzierte Immu- } \\
\text { nität gegen HBV }\end{array}$ & 266 & $69,1(62,8-74,7)$ & 1022 & $18,6(17,2-20,2)$ \\
\hline $\begin{array}{l}\text { Suszeptibilität gegen- } \\
\text { über HBV }\end{array}$ & 116 & $26,9(21,4-33,2)$ & 4543 & $77,1(75,4-78,8)$ \\
\hline \multicolumn{5}{|c|}{$\begin{array}{l}\text { Einen Überblick über die eingeschlossenen „Berufe im Gesundheitswesen“ gibt Onlinematerial 1. Die } \\
\text { Ergebnisse werden als ungewichtete Fallzahlen und gewichtete Prävalenzen mit 95-\%-Konfidenzinter- } \\
\text { vall (KI) berichtet. Gewichtungsfaktoren wurden eingesetzt, um für Non-Response und für Abweichun- } \\
\text { gen von der Zielpopulation in Bezug auf Alter, Geschlecht, Region, Staatsangehörigkeit, Gemeindegrö-- } \\
\text { Be und Bildungsstand zu korrigieren [19]. } n_{\text {ungewichtet gesamt }}=6202\end{array}$} \\
\hline
\end{tabular}

\section{Suszeptibilität}

Bei Männern war der Anteil suszeptibler Personen signifikant höher (74,6\%; $95 \%$ KI 72,4-76,7) als bei Frauen (69,4\%; $95 \%-K I$ 67,3-71,4). Die höchste Prävalenz suszeptibler Personen fand sich in den Geburtsjahrgängen 1937-1940, die niedrigste in den Geburtsjahrgängen 1989-1993 (88,8\%; 95\%-KI 85,3-91,5 vs. $32,9 \%$; $95 \%$-KI 27,5-38,7; • Abb. 1).

\section{Diskussion}

Basierend auf einem bevölkerungsrepräsentativen Survey aus Deutschland, einem Land mit niedriger HBsAg-Prävalenz, wurden in dieser Arbeit mögliche soziodemografische Determinanten der HBV-Infektion und impfinduzierten Immunität bei Männern und Frauen im Alter von 18-79 Jahren untersucht. In dieser Querschnittuntersuchung zeigte sich eine gegensätzliche Assoziation von stattgehabter HBV-Infektion und impfinduzierter Immunität mit den meisten der untersuchten Determinanten: Während die stattgehabte HBV-Infektion mit niedrigem Einkommen (Männer) und niedriger Bildung (Frauen) assoziiert war, waren höheres Einkommen und Bildung wichtige Determinanten der impfinduzierten Immunität bei beiden Geschlechtern. Teilnehmende der ersten Migrationsgeneration hatten eine signifikant erhöhte Chance für das Vorliegen einer stattgehabten HBV-Infektion, gleichzeitig war die Chance für das Vorliegen einer impfinduzierten Immunität bei Frauen der ersten Migrationsgeneration signifi- kant geringer als bei Frauen ohne Migrationshintergrund.

Im Jahr 1995 wurde in Deutschland die allgemeine Impfempfehlung für Kinder und Jugendliche ausgesprochen. Bereits die Geburtsjahrgänge (1981-1984) wiesen eine signifikant höhere Prävalenz der impfinduzierten Immunität auf als die vorhergehenden Geburtsjahrgänge 1977-1980, die zum Zeitpunkt der Impfempfehlung bereits im Jugendalter waren. Allerdings lag die Prävalenz der impfinduzierten Immunität bei den späteren Geburtsjahrgängen (1989-1993) mit maximal $67,1 \%$ weiterhin deutlich unter dem von der WHO definierten Impfquotenziel von $\geq 95 \%$ [2]. Möglicherweise ist dies dadurch zu erklären, dass auch die Teilnehmenden dieser Jahrgänge zum Zeitpunkt der Impfempfehlung 1995 bereits älter waren als der im Routineimpfschema für die Grundimmunisierung von Kindern angegebene Altersbereich. Bei ihnen hätte eine HBV-Impfung daher als Nachholimpfung (Catch-up-Impfung) durchgeführt werden müssen. Es ist bekannt, dass Nachholimpfungen seltener in Anspruch genommen werden als Standardimpfungen, die im Rahmen eines aktuell für das jeweilige Alter gültigen Impfkalenders angeboten werden [31].

Wie bereits in anderen bevölkerungsbasierten Surveys war auch in unserer Studie die Zugehörigkeit zur ersten Migrationsgeneration ein bedeutender Faktor für eine stattgehabte HBV-Infektion [11-15]. In einer französischen Studie variierte das Risiko einer HBV-Infektion bei Menschen der ersten Migrations- generation mit der HBsAg-Prävalenz im Herkunftsland [12]. Die erhobenen Angaben zu den Herkunftsländern der Teilnehmenden in DEGS1 waren aus methodischen Gründen nicht auswertbar. Aufgrund einer Analyse der Staatsangehörigkeiten der Teilnehmenden [30] ist jedoch davon auszugehen, dass die Stichprobe Teilnehmende aus Ländern mit niedriger, mittlerer und hoher HBsAg-Prävalenz enthielt. Das Risiko einer HBV-Infektion wurde daher vermutlich für Menschen aus Ländern mit hoher HBsAg-Prävalenz unterschätzt bzw. für Menschen aus Ländern mit niedriger HBsAg-Prävalenz überschätzt.

Trotz einer niedrigen HBsAg-Prävalenz im Aufnahmeland haben Menschen der ersten Migrationsgeneration auch hier in Deutschland ein erhöhtes Risiko, sich mit HBV zu infizieren, da meist enge Kontakte $\mathrm{zu}$ anderen Menschen mit Migrationserfahrung und dem Herkunftsland bestehen [14, 32, 33]. Sie sind daher eine wichtige Zielgruppe der HBV-Impfung. Der Zugang zu Präventionsmaßnahmen kann für Menschen der ersten Migrationsgeneration aufgrund von sprachlichen und kulturellen Barrieren erschwert sein [34]. So ergab eine frühere Auswertung des DEGS1, dass Frauen der ersten Migrationsgeneration über präventive Gesundheitsangebote weniger informiert waren und diese auch seltener in Anspruch nahmen als Frauen ohne Migrationshintergrund [29]. Da auch in unseren Auswertungen Frauen der ersten Migrationsgeneration eine geringere Chance für eine impfinduzierte Immunität hatten als Frauen ohne Migrationshintergrund, sollten die besonderen Bedarfe des Zugangs zu Impfung und Prävention für diese Gruppe weiter untersucht und entsprechende Maßnahmen umgesetzt werden.

In den über schrittweise Vorwärtsselektion gebildeten multivariablen Analysen, in der alle in univariablen Analysen identifizierten Faktoren untersucht wurden, konnte eine Assoziation der HBVInfektion bei Männern unabhängig mit einem niedrigen Einkommen und bei Frauen unabhängig mit einer niedrigen Bildung beobachtet werden. Da Einkommen, Bildung und Migrationsstatus eng miteinander korrelieren, haben wir 


\section{Leitthema}

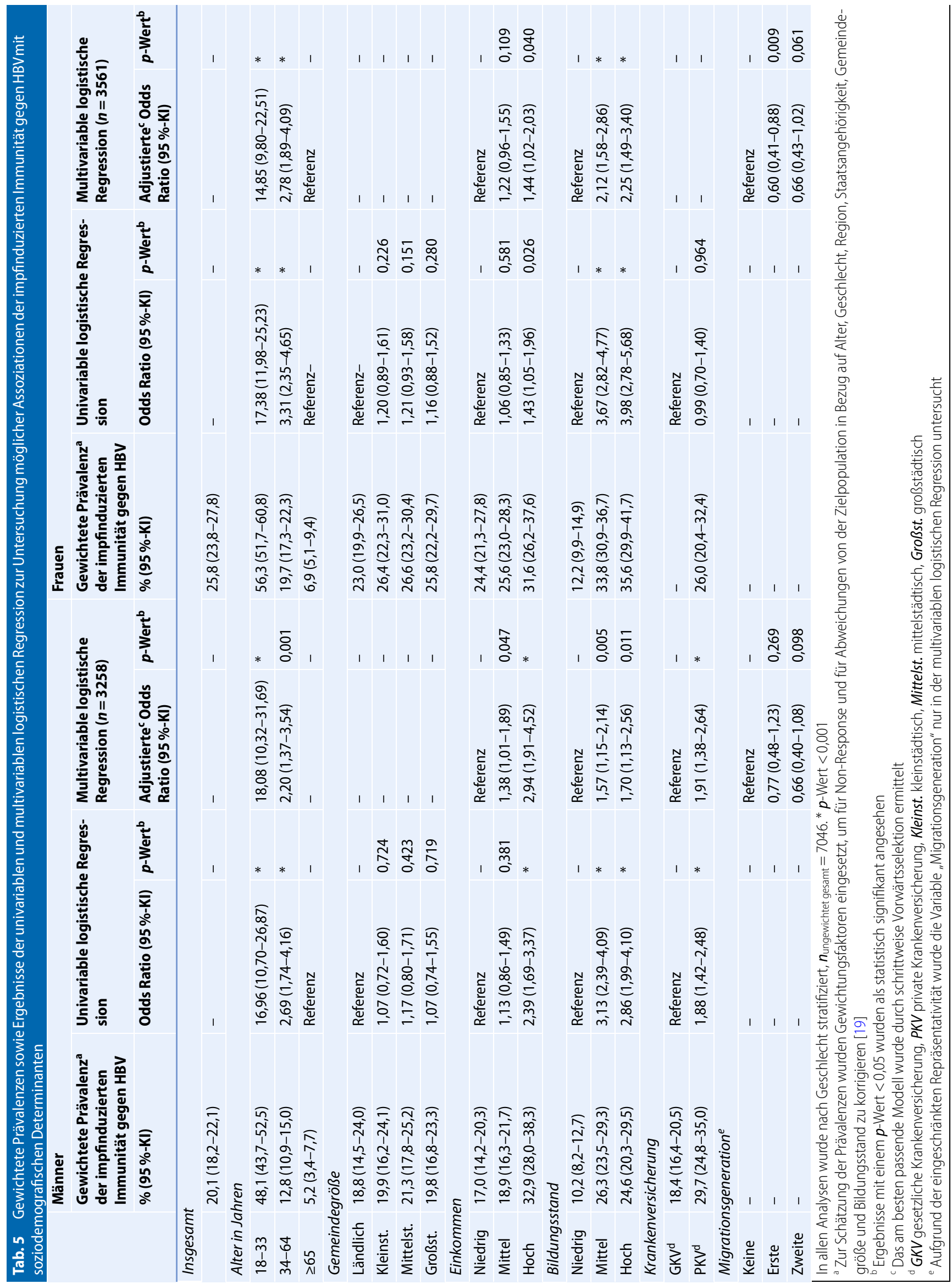


in zusätzlichen explorativen, multivariablen Analysen differenziert für Männer und Frauen Interaktionsterme eingeschlossen (Ergebnisse nicht gezeigt). Frauen mit niedrigem Einkommen und niedriger Bildung hatten - anders als Männer - geringfügig höhere Chancen einer HBV-Infektion. Eine weitere Interaktion konnte für Frauen zwischen der ersten Einwanderungsgeneration und niedriger Bildung sowie für Männer zwischen der ersten Einwanderungsgeneration und niedrigem Einkommen festgestellt werden.

In anderen bevölkerungsbasierten Studien war die stattgehabte HBV-Infektion ebenfalls mit einem niedrigen Einkommen bzw. materieller Armut assoziiert [12, 15] und zeigte widersprüchliche Ergebnisse bezüglich der Assoziation mit dem Bildungsstand [12, 15]. Allerdings war ein niedriger sozioökonomischer Status in einer französischen Studie auch mit weiteren Risikofaktoren der HBV-Infektion, wie der Herkunft aus Ländern mit erhöhter HBsAg-Prävalenz, Tätowierungen, Drogengebrauch oder früherer Inhaftierung, assoziiert [12]. Ein Confounding durch Einflussgrößen, die in DEGS1 nicht erfasst wurden, ist somit nicht auszuschließen.

Internationale Studien zeigen, dass ein höherer Bildungsstand mit einem höheren Wissensstand zur HBV-Infektion, deren Risiken und Präventionsmaßnahmen einhergeht $[12,35,36]$. In unserer Studie war die impfinduzierte Immunität bei beiden Geschlechtern unabhängig mit mittlerer und hoher Bildung assoziiert und bestätigte die Ergebnisse anderer Untersuchungen [15, 37, 38].

Auch ein hohes Einkommen war bei Männern und Frauen unabhängig mit dem Vorliegen einer impfinduzierten Immunität assoziiert. Eine mögliche Erklärung hierfür könnten die Regelungen für eine Kostenübernahme der Impfungen sein: Grundsätzlich werden in Deutschland alle durch die STIKO empfohlenen Impfungen von der GKV übernommen [39]. Ausnahmen sind Impfungen aufgrund eines berufsassoziierten Risikos, die vom Arbeitgeber bezahlt werden, und Impfungen in Vorbereitung privater (Fern-)Reisen [7]. Diese mussten bis 2007 stets selbst bezahlt werden [7]. Da in einem Telefon-Survey und in einer Studie unter Blutspendenden „private Reisen“ als eine der Hauptindikationen für eine Hepatitis-B-Impfung genannt wurden [37, 38], könnte ein hoher Anteil an Reiseimpfungen die Assoziation der impfinduzierten Immunität mit höherem Einkommen erklären.

Eine private Krankenversicherung bietet umfangreichere Leistungspakete und höhere Honorare für Ärztinnen und Ärzte als die GKV, setzt bei Angestellten aber das Überschreiten einer bestimmten Einkommensgrenze voraus [39]. 2012 waren $11 \%$ der deutschen Bevölkerung privat krankenversichert [39]. In dieser Studie war die Chance für das Vorliegen einer impfinduzierten Immunität bei Männern mit privater Krankenversicherung fast doppelt so hoch wie bei Männern mit gesetzlicher Krankenversicherung. Bei Frauen hingegen fand sich diese Assoziation nicht; allerdings waren Frauen auch seltener privat krankenversichert. Da die Frage der Kostenerstattung nach Angabe von befragten Ärztinnen und Ärzten eine mögliche Barriere für Impfungen ist [40], könnte die Zugehörigkeit zur PKV das Angebot und die Inanspruchnahme der HBV-Impfung begünstigen.

Das Risiko einer HBV-Infektion ist bei medizinischem Personal erhöht, z. B. durch häufigen Kontakt zu Körperflüssigkeiten und Stichverletzungen. Für Gesundheitspersonal in Deutschland besteht deshalb seit Beginn der 1980erJahre eine HBV-Impfempfehlung einschließlich einer Auffrischungsimpfung bei sinkendem Anti-HBs-Titer [41], sodass neue Meldungen berufsassoziierter HBV-Infektionen heutzutage selten sind [42].

In dieser Studie untersuchten wir ausgewählte medizinische Berufsgruppen, die direkten Patientenkontakt und ein erhöhtes Risiko für eine Exposition gegenüber potenziell infektiösen Körperflüssigkeiten haben. Teilnehmende mit einem Beruf im Gesundheitswesen wiesen keine höhere Prävalenz stattgehabter HBV-Infektionen auf als Teilnehmende ohne medizinischen Beruf. Die Prävalenz der impfinduzierten Immunität war hingegen bei Teilnehmenden mit einem Beruf im Gesundheitswesen signifikant höher. Interessanterweise wiesen ca. 30 \% der Teilnehmenden mit einem medizinischen Beruf keine impfinduzierte Immunität gegen $\mathrm{HBV}$ auf und fast $27 \%$ waren für eine HBV-Infektion suszeptibel. Hierbei ist zu berücksichtigen, dass die Indikation für eine HBV-Impfung immer auf einer individuellen, arbeitsplatzbezogenen Risikobeurteilung beruht. In bestimmten Fällen (wie z.B. bei einer reinen Verwaltungstätigkeit) mag daher auch bei Personen mit medizinischen Berufen keine Indikation zur HBV-Impfung bestehen. Auch ist die HBV-Impfung für Gesundheitspersonal nicht verpflichtend.

\section{Stärken und Limitationen}

Die Repräsentativität der DEGS1-Daten für die deutsche Allgemeinbevölkerung ist hoch und die Teilnahmerate der neu gezogenen Stichprobe mit anderen europäischen Surveys vergleichbar [19, 43]. Allerdings liegt der Beginn der Datenerhebung bereits mehr als 10 Jahre zurück. Obwohl die Daten aus DEGS1 absehbar noch für einige Jahre die aktuellste bevölkerungsbasierte Datenbasis für die untersuchten Fragestellungen darstellen, können sich die aktuellen Prävalenzen und Zusammenhänge in der Zwischenzeit verändert haben.

Zur Minimierung möglicher Verzerrungen durch selektives Teilnahmeverhalten kamen Non-ResponderAnalysen, Vergleiche mit der Zielpopulation und Gewichtungsfaktoren zum Einsatz [19, 20]. In DEGS1 wurden allerdings nur Personen eingeschlossen, die außerhalb von Institutionen, wie z.B. Flüchtlingseinrichtungen oder Gefängnissen, lebten [19]. Bestimmte Risikogruppen der HBV-Infektion, wie Inhaftierte oder Flüchtlinge [1, 44], wurden daher nicht untersucht. Auch waren die Auswertungen für Menschen mit Migrationshintergrund nur mit Einschränkungen möglich [29, 30].

Das Vorliegen einer impfinduzierten Immunität bzw. einer stattgehabten HBV-Infektion wurde serologisch ermittelt. Es wird angenommen, dass ein AntiHBs-Titer von > 10 IU/l einen langfristigen Schutz vor einer Hepatitis B bietet. Allerdings sprechen ca. $5 \%$ der gesunden 
Erwachsenen nicht auf die Hepatitis-BImpfung an und auch nach erfolgreicher Impfung fällt der Anti-HBs-Titer nach 4-10 Jahren bei bis zu $50 \%$ der gesunden Erwachsenen auf niedrige oder nicht mehr nachweisbare Spiegel [45-49]. Die tatsächliche Impfquote wurde daher vermutlich in dieser Studie unterschätzt. Hierfür spricht auch, dass laut Auswertung der Daten auf Basis der vorgelegten Impfbücher und der Selbstauskünfte 32,9\% der DEGS1-Teilnehmenden mindestens eine HepatitisB-Impfung erhalten hatten [50], aber nur $22,9 \%$ eine serologisch nachweisbare impfinduzierte Immunität aufwiesen [4].

In dieser Studie werden stattgehabte HBV-Infektionen, die z.T. bereits vor Jahrzehnten und/oder außerhalb Deutschlands erworben wurden, als Zeichen für ein aktuell erhöhtes Infektionsrisiko verwendet. Da sich z.B. die soziodemografischen Merkmale der Betroffenen seit der Infektion verändert haben könnten, sollte dies bei der Interpretation der Ergebnisse berücksichtigt werden.

\section{Fazit}

Obwohl die zugrunde liegenden Daten bereits vor rund einer Dekade erhoben wurden, können die Ergebnisse dieser Studie dazu beitragen, die HBV-Prävention in Deutschland zielgenauer auszurichten.

Zwar legt die steigende Prävalenz der impfinduzierten Immunität in den jüngeren Altersgruppen nahe, dass die Impfung die HBV-Transmission in der Zukunft begrenzen wird. Um das Ziel der Elimination der Virushepatitis bis $2030 \mathrm{zu}$ erreichen, sind jedoch weitere Maßnahmen erforderlich, z.B. um Impflücken zu schließen.

Die Ergebnisse dieser Studie liefern Hinweise, dass Präventionsstrategien insbesondere die Bedarfe von Menschen mit niedrigem Bildungsstand und eigener Migrationserfahrung berücksichtigen sollten. Auch könnten evtl. die Regelungen der Kostenübernahme der HBV-Impfung durch die Krankenkassen von Bedeutung sein. Um diese und weitere mögliche Maßnahmen, wie eine kostenlose, mehrsprachige und aufsuchende Impfberatung und Testung, auszurichten, sollten die Ergebnisse dieser Studie aktualisiert und präzisiert werden.

\section{Korrespondenzadresse}

\section{Dr. Christina Poethko-Müller, MSC}

Abteilung für Epidemiologie und Gesundheitsmonitoring, FG 25 Körperliche Gesundheit, Robert Koch-Institut

General-Pape-Straße 62-66, 12101 Berlin, Deutschland

poethko-muellerc@rki.de

Danksagung. Diese Studie hätte nicht realisiert werden können ohne die Unterstützung von vielen Kolleginnen und Kollegen innerhalb des Robert Koch-Instituts. Wir danken insbesondere den Studienteams für ihre exzellente Arbeit und ihr großes Engagement während der dreijährigen Datenerhebung. Wir danken außerdem den Bürgermeisterinnen, Bürgermeistern und Verwaltungen der 180 ausgewählten Gemeinden für ihre Unterstützung der Feldlogistik.

Förderung. Die Studie wurde mit Mitteln des Robert Koch-Instituts und des Bundesministeriums für Gesundheit finanziert.

Funding. Open Access funding enabled and organized by Projekt DEAL.

\section{Einhaltung ethischer Richtlinien}

Interessenkonflikt. A. Brodzinski, A. Neumeyer-Gromen, S. Dudareva, R. Zimmermann, U. Latza, V. Bremer und C. Poethko-Müller geben an, dass kein Interessenkonflikt besteht.

Alle beschriebenen Untersuchungen am Menschen oder an menschlichem Gewebe wurden mit Zustimmung der zuständigen Ethikkommission, im Einklang mit nationalem Recht sowie gemäß der Deklaration von Helsinki von 1975 (in der aktuellen, überarbeiteten Fassung) durchgeführt. Das Studienprotokoll war von den Bundes- und Landesbeauftragten für den Datenschutz sowie von der Ethikkommission der Charité - Universitätsmedizin Berlin (Antragsnummer EA2/047/08) bewilligt worden. Von allen beteiligten Patienten liegt eine Einverständniserklärung vor.

Open Access. Dieser Artikel wird unter der Creative Commons Namensnennung 4.0 International Lizenz veröffentlicht, welche die Nutzung, Vervielfältigung, Bearbeitung, Verbreitung und Wiedergabe in jeglichem Medium und Format erlaubt, sofern Sie den/die ursprünglichen Autor(en) und die Quelle ordnungsgemäß nennen, einen Link zur Creative Commons Lizenz beifügen und angeben, ob Änderungen vorgenommen wurden.

Die in diesem Artikel enthaltenen Bilder und sonstiges Drittmaterial unterliegen ebenfalls der genannten Creative Commons Lizenz, sofern sich aus der Abbildungslegende nichts anderes ergibt. Sofern das betreffende Material nicht unter der genannten Creative
Commons Lizenz steht und die betreffende Handlung nicht nach gesetzlichen Vorschriften erlaubt ist, ist für die oben aufgeführten Weiterverwendungen des Materials die Einwilligung des jeweiligen Rechteinhabers einzuholen.

Weitere Details zur Lizenz entnehmen Sie bitte der Lizenzinformation auf http://creativecommons.org/ licenses/by/4.0/deed.de.

\section{Literatur}

1. World Health Organization (2020) Fact sheet hepatitis B. https://www.who.int/news-room/ fact-sheets/detail/hepatitis-b. Zugegriffen: 28. Juni 2021

2. World Health Organization (2016) Global health sector strategy on viral hepatitis 2016-2021. https://apps.who.int/iris/bitstream/ handle/10665/246177/WHO-HIV-2016.06-eng. pdf? sequence=1\&isAllowed=y. Zugegriffen: 3 . Juli 2021

3. Bundesministerium für Gesundheit, Bundesministerium für Wirtschaftliche Zusammenarbeit und Entwicklung (2016) BIS 2030 - Strategie zur Eindämmung von HIV, Hepatitis B und C und anderen sexuell übertragbaren Infektionen. https://www.bundesgesundheitsministerium.de/ fileadmin/Dateien/5_Publikationen/Praevention/ Broschueren/Strategie_BIS_2030_HIV_HEP_STI. pdf.Zugegriffen:4.0kt. 2021

4. Poethko-Müller C, Zimmermann R, Hamouda O et al (2013) Die Seroepidemiologie der Hepatitis A, B und $C$ in Deutschland - Ergebnisse der Studie zur Gesundheit Erwachsener in Deutschland (DEGS1). Bundesgesundheitsblatt Gesundheitsforschung Gesundheitsschutz 56:707-715. https://doi.org/ 10.1007/s00103-013-1673-x

5. Lernout T, Hendrickx G, Vorsters A, Mosina L, Emiroglu N, Van Damme P (2014) A cohesive European policy for hepatitis $B$ vaccination, are we there yet? Clin Microbiol Infect 20(5):19-24

6. Ott JJ, Stevens GA, Groeger J, Wiersma ST (2012) Global epidemiology of hepatitis B virus infection: new estimates of age-specific $\mathrm{HBsAg}$ seroprevalence and endemicity. Vaccine 30:2212-2219. https://doi.org/10.1016/j.vaccine. 2011.12.116

7. Robert Koch-Institut (1972-2019) Archiv der STIKO-Empfehlungen. https://www.rki.de/DE/ Content/Kommissionen/STIKO/Empfehlungen/ Archiv/seit_1972/archiv_tab.html.Zugegriffen: 3 . Juli 2021

8. Sperle I, Steffen G, Leendertz SA et al (2020) Prevalence of hepatitis B, C, and D in Germany: results from a scoping review. Front Public Health 8:424.https://doi.org/10.3389/fpubh.2020.00424

9. Steffen G, Sperle I, Harder T et al (2021) Hepatitis $B$ vaccination coverage in Germany: systematic review. Research square. https://doi.org/10. 21203/rs.3.rs-311330/v1.Zugegriffen: 3. Juli 2021

10. von Laer A, Simeonova Y, Harder T, Zimmermann R, Dudareva-Vizule S (2017) Virushepatitis B und Dim Jahr 2016. Epidemiol Bull 31:297-308

11. Rotermann M, Langlois K, Andonov A, Trubnikov M (2013) Seroprevalence of hepatitis B and C virus infections: results from the 2007 to 2009 and 2009 to 2011 Canadian health measures survey. Health Rep 24:3-13

12. Meffre C, Le Strat Y, Delarocque-Astagneau E et al (2010) Prevalence of hepatitis B and hepatitis C virus infections in France in 2004: social factors are important predictors after adjusting for known risk 
factors. J Med Virol 82:546-555. https://doi.org/10. 1002/jmv.21734

13. Wasley A, Kruszon-Moran D, Kuhnert Wet al (2010) The prevalence of hepatitis $B$ virus infection in the United States in the era of vaccination. J Infect Dis 202:192-201.https://doi.org/10.1086/653622

14. Hahné SJ, De Melker HE, Kretzschmar $M$ et al (2012) Prevalence of hepatitis B virus infection in The Netherlands in 1996 and 2007. Epidemiol Infect 140:1469-1480. https://doi.org/10.1017/ S095026881100224X

15. Kim HS, Rotundo L, Yang JD et al (2017) Racial/ ethnic disparities in the prevalence and awareness of hepatitis B virus infection and immunity in the United States. J Viral Hepat 24:1052-1066. https:// doi.org/10.1111/jvh.12735

16. Lampert T, Kroll L, Müters S, Stolzenberg H (2013) Messung des sozioökonomischen Status in der Studie zur Gesundheit Erwachsener in Deutschland (DEGS1). Bundesgesundheitsblatt Gesundheitsforschung Gesundheitsschutz 56:631-636. https://doi.org/10.1007/s00103-012-1663-4

17. Lu PJ, Rodriguez-Lainz A, O'halloran A, Greby S, Williams WW (2014) Adult vaccination disparities among foreign-born populations in the U.S., 2012. Am J Prev Med 47:722-733. https://doi.org/10. 1016/j.amepre.2014.08.009

18. Oster AM, Sternberg M, Nebenzahl S et al (2014) Prevalence of HIV, sexually transmitted infections, and viral hepatitis by urbanicity, among men who have sex with men, injection drug users, and heterosexuals in the United States. Sex Transm Dis 41:272-279. https://doi.org/10.1097/OLQ. 0000000000000110

19. Kamtsiuris P, Lange M, Hoffmann R et al (2013) Die erste Welle der Studie zur GesundheitErwachsener in Deutschland (DEGS1): Stichprobendesign, Response, Gewichtung und Reprasentativität. Bundesgesundheitsblatt Gesundheitsforschung Gesundheitsschutz 56:620-630. https://doi.org/ 10.1007/s00103-012-1650-9

20. Scheidt-Nave C, Kamtsiuris $P$, Gößwald $A$ et al (2012) German health interview and examination survey for adults (DEGS) — design, objectives and implementation of the first data collection wave. BMC Public Health 12:730. https://doi.org/10. 1186/1471-2458-12-730

21. Gößwald A, Lange M, Dölle R, Hölling H (2013) Die ersteWelle derStudiezur GesundheitErwachsener in Deutschland (DEGS1): Gewinnung von Studienteilnehmenden, Durchfuhrung der Feldarbeit und Qualitatsmanagement. Bundesgesundheitsblatt Gesundheitsforschung Gesundheitsschutz 56:611-619. https://doi.org/10.1007/s00103013-1671-z

22. Bundesministerium für Arbeit und Soziales (2011) Aktualisierung der Berichterstattung über die Verteilung von Einkommen und Vermögen in Deutschland. https://www.armuts-undreichtumsbericht.de/SharedDocs/Downloads/ Berichte/aktualisierung-berichterstattung-uberverteilung.pdf?_blob=publicationFile \&v $=5 . \mathrm{Zu}-$ gegriffen: 3. Juli 2021

23. Statistisches Bundesamt (2013) Armut und soziale Ausgrenzung in Deutschland und der Europäischen Union. Ergebnisse aus LEBEN IN EUROPA (EU-SILC) 2021. https://www.destatis.de/ DE/Methoden/WISTA-Wirtschaft-und-Statistik/ 2013/12/armut-soziale-ausgrenzung-122013. pdf?_blob=publicationFile. Zugegriffen: 3. Juli 2021

24. Brauns H, Scherer S, Steinmann S (2003) The CASMIN educational classification in international comparative research. In: Hoffmeyer-Zlotnik J,
Wolf C (Hrsg) Advances in cross-national comparison. Kluwer, New York, S 221-244

25. Brauns H, Steinmann S (1999) Educational reform in France, west-Germany and the United Kingdom: updating the CASMIN educational classification. ZUMA-Nachrichten, S7-44

26. Bundesagentur für Arbeit (2013) Klassifikation der Berufe 2010 - Systematisches Verzeichnis. https:// statistik.arbeitsagentur.de/Statischer-Content/ Grundlagen/Klassifikationen/Klassifikation-derBerufe/KIdB2010/Systematik-Verzeichnisse/ Generische-Publikationen/SystematischesVerzeichnis-Berufsbenennung.xls. Zugegriffen: 28. Juni 2021

27. Haussig JM, Nielsen S, Gassowski M et al (2018) A large proportion of people who inject drugs are susceptible to hepatitis B: results from a biobehavioural study in eight German cities. Int J Infect Dis 66:5-13. https://doi.org/10.1016/j.ijid. 2017.10.008

28. Jansen K, Thamm M, Bock CT et al (2015) High prevalence and high incidence of coinfection with hepatitis $B$, hepatitis $C$, and syphilis and low rate of effective vaccination against hepatitis B in HIV positive men who have sex with men with known date of HIV seroconversion in Germany. PLoS ONE 10:e142515. https://doi.org/10.1371/journal. pone.0142515

29. Rommel A, Saß AC, Born S, Ellert U (2015) Die gesundheitliche Lage von Menschen mit Migrationshintergrund und die Bedeutung des sozioökonomischen Status : Erste Ergebnisse der Studie zu Gesundheit Erwachsener in Deutschland (DEGS1) Bundesgesundheitsblatt Gesundheitsforschung Gesundheitsschutz 58:543-552. https://doi.org/ 10.1007/s00103-015-2145-2

30. Saß AC, Grüne B, Brettschneider AK, Rommel A, Razum O, Ellert U (2015) Beteiligung von Menschen mit Migrationshintergrund an Gesundheitssurveys des Robert Koch-Instituts. Bundesgesundheitsblatt Gesundheitsforschung Gesundheitsschutz 58:533-542. https://doi.org/ 10.1007/s00103-015-2146-1

31. Poethko-Müller C, Kuhnert R, Schlaud M (2007) Durchimpfung und Determinanten des Impfstatus in Deutschland. Ergebnisse des Kinder- und Jugendgesundheitssurveys (KiGGS). Bundesgesundheitsblatt Gesundheitsforschung Gesundheitsschutz 50:851-862. https://doi.org/10.1007/ s00103-007-0248-0

32. Hahné SJ, Veldhuijzen IK, Smits LJ, Nagelkerke N, Van De Laar MJ (2008) Hepatitis B virus transmission in The Netherlands: a population-based, hierarchical case-control study in a very lowincidence country. Epidemiol Infect 136:184-195 https://doi.org/10.1017/S0950268807008205

33. Diel R, Helle J, Gottschalk R (2005) Transmission of hepatitis B in Hamburg, Germany, 1998-2002: a prospective, population-based study. Med Microbiol Immunol 194:193-199. https://doi.org/ 10.1007/s00430-005-0237-6

34. Spallek J, Zeeb H, Razum O (2010) Prevention among immigrants: the example of Germany. BMC Public Health 10:92. https://doi.org/10.1186/ 1471-2458-10-92

35. Lu PJ, O'halloran A, Kennedy ED et al (2017) Awareness among adults of vaccine-preventable diseases and recommended vaccinations, United States, 2015. Vaccine 35:3104-3115. https://doi. org/10.1016/j.vaccine.2017.04.028

36. GanczakM,Dmytrzyk-Danilow G, Korzen M, DrozdDabrowska M, Szych Z (2016) Prevalence of HBV infection and knowledge of hepatitis $B$ among patients attending primary care clinics in Poland.
J Community Health 41:635-644. https://doi.org/ 10.1007/s10900-015-0139-5

37. Schenkel K, Radun D, Bremer V, Bocter N, Hamouda O (2008) Viral hepatitis in Germany: poor vaccination coverage and little knowledge about transmission in target groups. BMC Public Health 8:132. https://doi.org/10.1186/1471-2458-8-132

38. Ringwald J, Mertz I, Zimmermann R et al (2006) Hepatitis $B$ vaccination status among healthy adults in Germany. Health Policy 79:306-312. https://doi.org/10.1016/j.healthpol.2006.01.012

39. Busse R, Blümel M (2014) Germany: health system review. Health Syst Transit 16:1-296

40. Neufeind J, Betsch C, Habersaat KB, Eckardt M, Schmid P, Wichmann O (2020) Barriers and drivers to adult vaccination among family physicians - insights for tailoring the immunization program in Germany. Vaccine 38:4252-4262. https://doi.org/10.1016/j.vaccine.2020.04.052

41. Jilg W (1995) Selective risk group strategies in Europe. Vaccine 13(1):S44-46. https://doi.org/10. 1016/0264-410x(95)80049-j

42. Dudareva S, Kremer K, Harder T, Maisa A, Bremer V Zimmermann R (2020) Virushepatitis B und D im Jahr 2019. Epidemiol Bull 30:3-17

43. Tolonen H, Koponen P, Aromaa A et al (2008) Review of health examination surveys in Europe. National Public Health Institute, Helsinki

44. Hampel A, Solbach P, Cornberg M, Schmidt RE, Behrens GM, Jablonka A (2016) AktuelleSeroprävalenz, Impfstatus und prädiktiver Wert der Leberenzyme für Hepatitis B bei Flüchtlingen in Deutschland. Bundesgesundheitsblatt Gesundheitsforschung Gesundheitsschutz 59:578-583. https://doi.org/ 10.1007/s00103-016-2333-8

45. Coates T, Wilson R, Patrick G, Andre F, Watson V (2001) Hepatitis B vaccines: assessment of the seroprotective efficacy of two recombinant DNA vaccines. Clin Ther 23:392-403. https://doi.org/10. 1016/s0149-2918(01)80044-8

46. Cornberg M, Protzer U, Petersen J et al (2011) Prophylaxis, diagnosis and therapy of hepatitis $B$ virus infection-the German guideline. $Z \mathrm{Ga}$ stroenterol 49:871-930. https://doi.org/10.1055/ s-0031-1273462

47. Jilg W, Schmidt M, Deinhardt F (1989) Fouryear experience with a recombinant hepatitis $B$ vaccine. Infection 17:70-76. https://doi.org/10. 1007/BF01646879

48. Wainwright RB, Bulkow LR, Parkinson AJ, Zanis C, Mcmahon BJ (1997) Protection provided by hepatitis B vaccine in a Yupik Eskimo population - results of a 10-year study.JInfectDis 175:674-677. https:// doi.org/10.1093/infdis/175.3.674

49. Cornberg M, Protzer U, Petersen J et al (2011) Aktualisierung der S 3-Leitlinie zur Prophylaxe, Diagnostik und Therapie der Hepatitis-B-Virusinfektion. Z Gastroenterol 49:871-930. https://doi. org/10.1055/s-0031-1273462

50. Poethko-Müller C, Schmitz R (2013) Impfstatus von Erwachsenen in Deutschland: Ergebnisse der Studie zur Gesundheit Erwachsener in Deutschland (DEGS1). Bundesgesundheitsblatt Gesundheitsforschung Gesundheitsschutz 56:845-857. https://doi.org/10.1007/s00103-013-1693-6 